\title{
Prosthodontic Management of Special (Severely III) Patients
}

\author{
Dr. Isha Rastogi \\ MDS Prosthodontics Address- 2/9 lajpat Nagar, chowk, lucknow, up, India \\ Mayo Institute of Medical Sciences and Hospital, Barabanki.
}

\begin{abstract}
Prosthodontics is a wonderful branch.It trains us to deal with various kinds of patients. Sometimes we face very special patients, namely critical patients. Comatose and severely ill patients suffer a multiple number of difficulties.Their oral care is of paramount importance because they are dependent on their family and attendants.A Prosthodontist is often sought after to manage these patients.One's knowledge and clinical skills are utilized here to prepare adequate appliances for them.These appliances are made with ease and provide oral hygiene for these special patients. The call of the hour for the Prosthodontist is to understand and develop a determination to treat such patients successfully.
\end{abstract}

Keywords: comatose; critical; special; Prosthodontist; care

\section{Introduction}

Hospital admissions of patients in coma are more frequent than might be expected by those not regularly active in caring for them(1). Prevalence of trauma to oral tissues in the comatose patient is not well documented, but when it occurs it is quite destructive and distressful for both patient and family members.(2)

Regular oral assessment and individualised oral care, along with the use of a standardised protocol for oral care (incorporating proven modalities) is vital for optimal oral care in the critically ill patient(3). Such severely/critically ill patients have a higher risk of oral disease due to compromised oral hygiene as a consequence of their impairment, oral manifestations of their particular condition and/or the side effects of drug regimes, notably xerostomia and sugar in medicines(4).

Thus Dental and Prosthodontic care plays an important role in the multidisciplinary approach, which is used in palliative and long-term care to address the complex needs of terminally ill patients(5).These patients necessitate extra knowledge and care to prevent potential complications causing otherwise unnecessary morbidity and mortality(6).

\section{Problems}

The neurophysiology of jaw movement in the comatose patient is the basis for the design of an intraoral prosthesis $(10)$. which prevents discoordinate or neuropathologic mandibular chewing movements and facilitates healing of pre-existing lesions(11).

Neuropathological chewing in hospitalized, comatose patients can occur from severe brain damage following a closed head injury, hypoxia, and septic shock.(7) Also ruminatory reflex chewing activities in the comatose patient and the associated prostheses used in their treatment have been well documented in the literature(13).

Trauma to oral soft tissues in comatose patients may be more widespread than reported, as no extensive study of this problem has been conducted. Various appliances for the prevention of self-inflicted injuries to oral tissues particularly in children and the physically and mentally challenged have been documented, but there is little information on their use in adult comatose patients. Because comatose patients lack cerebral control of the masticatory cycle, they can easily injure themselves. Although it is not uncommon for patients with a decreased level of consciousness and in need of intensive care to be restrained to prevent injury due to involuntary movement of the limbs, head and neck restraint is often difficult and may be dangerous to the patient(2).

\section{Objectives(9)}

To provide a simple oral appliance to prevent damage to teeth or surrounding soft tissue, to provide an appliance that is resilient/flexible, that does not interfere with normal mandibular movements, that does not require the making of impressions or use of any special sedation, that can be easily done by either medically skilled/ unskilled attendants of patient and that permits regular oral hygiene and oral inspection.

\section{Management(12)}

The management of such trauma also varies depending on the medical history of the patient; the etiology of the behavior; and the severity, frequency, and method of inflicting injury. 
Materials(8)

Materials used for making these devices are polymers such as acrylic resins and metals like silver and stainless steel.

\section{Appliances/Methods(8)}

They are occlusal bite planes, mouth props, Padded tongue blades, intermaxillary fixation, mandibular cast silver caps with acrylic bite-blocks, stock fluoride trays, tongue guards / mouth guards and a modified retainer.

Occlusal bite planes are removable dental appliances carefully molded to fit the upper or lower arch of teeth. These bite planes are typically made of heat-cured acrylic resin. Soft acrylic or light cured composite, or vinyl splints may be made more quickly and cheaply, but are not durable, and hence indicated for short-term use. Soft splints are preferred in children to accommodate growth changes. Thy are used to correct an anterior crossbite(14).

Mouth Props are made of non elastic foam such as polyurethane, foamed polyethylene, foamed epoxy which help in retaining the mouth open. The block or prop is made with different cross sectional dimensions so that the desired mouth opening can be achieved without fatigue to the jaw muscles. When the teeth indent into the foam plastic material, the block is locked in place against any slippage and is disposable after use(8).They help prevent injury to the patient should the jaws suddenly be clenched.These might be easily dislocated in the mouth and should be securely attached to a length of dental floss for quick removal to prevent their aspiration by the patient(14).

Padded tongue blades are made by wrapping adhesive tape around tongue depressor blades. It is mostly used by health care providers during oral examination and while maintaining oral hygiene.It acts as a lever when it is used to correct anterior crossbite(14).

Intermaxillary fixation using arch wires in combination with hard acrylic devices was used to prevent neuropathologic chewing. This was more of a surgical management, at times painful for the patient and could not be removed immediately in case of emergency. If the wire or rigid plastic components break they create jagged, virtually nondetectable foreign bodies which could be aspirated into the lungs. These may also lacerate the throat, larnyx or soft tissue of the oral cavity(8).To ensure comfort and prevent aspiration, it is advisable to tape either a scissors or a wire cutter, and a hemostat to the bed for rapid removal of elastic or wire traction in case of vomiting. Use of a soft toothbrush and the water-pik gives very satisfactory results during intermaxillary fixation(15).

Stock fluoride trays were useful as an initial or interim device in the case of hospitalized unconscious paediatric patients with uncoordinated mandibular movements or clenching.

U-shaped oral appliance is formed from acrylic resin material.Typically, this is about $3 \mathrm{~mm}$ in thickness, although other thicknesses can be used. The appliance has a generally U-shaped body formed by an extra-oral leg and an intra-oral leg and a centrally curved contour in between the two. A periodic change of appliance position is desirable to prevent chronic soreness and cutting of the mouth. This was not possible with the prosthesis units used before. This appliance gives the feasibility of being easily switched from side-to-side which prevents excessive irritation to the corner of the mouth. This also prevents unilateral compression to the teeth and their supporting structures. Its insertion and removal is simple, therefore it can be easily used by either medically skilled or unskilled attendants of the patient (9).

Mouth guard/protector(14) -Although the protective benefits from the use of mouth protectors alone cannot be separated from the combined use of the face guard and mouth protector, the value of the latter in reducing tooth injuries can at least be implied.There are three general types:-stock/readymade, those formed directly in the mouth, custom made from a cast of the maxillary dental arch.

Conventional mouth guard is made using acrylic with adequate coverage of teeth to ensure good retention, but at the same time not overly extended to avoid impingement on soft tissue and muscle attachment. A long flexible handle made of stainless steel wire is attached to the anterior portion of the mouthguard(2). This could be secured to the patient's hospital gown with a safety pin to prevent accidental loss or possible inhalation of the appliance. The labial portion of the appliance is slightly thickened $(8 \mathrm{~mm})$ by layering to prevent the lips from becoming trapped between the anterior teeth. The appliance can be kept in the mouth at all times except when removed for daily oral hygiene procedures. The practice had been to construct either a full coverage mouthguard or one covering the anterior sextant using traditional materials; however, these materials lack proper adaptation to the dentition, hence were not adequately retained. Dislodgement of earlier appliances may cause even more serious complications if it is inhaled. It can be modified to make it, a simple unilateral arch appliance of acrylic. This design, which covered two-thirds of the mandibular occlusal surface, is preferred only for short term. In the long term, a full arch stent would be more appropriate to prevent an imbalance to the occlusion and inadvertent overeruption of teeth not covered by a partial appliance. 
One- piece occlusal guard was designed to prevent further trauma from the protrusion of the patient's tongue between the occlusal guard and the teeth. The thickness of this 1-piece occlusal guard was kept $15-20 \mathrm{~mm}$ at the incisors(2).This thickness also allows room for the suction tube. If the occlusal guard is too thick, it will strain the patient's facial muscles. The occlusal guard was designed to cover approximately 2-3 $\mathrm{mm}$ of the facial surfaces of the incisal edges of the teeth above the height of contour.The design incorporated coverage of the entire palatal surface of the teeth and 3-5 $\mathrm{mm}$ of the palatal soft tissue. The wax pattern was fabricated using pink modelling wax. A lingual extension of wax, $2 \mathrm{~mm}$ thick, was added to the pattern. It was placed 3-5 $\mathrm{mm}$ posterior to the mandibular incisal contact points and extended entirely down the lingual surfaces, from the mandibular left to right premolars, to prevent the tongue from moving forward. The wax pattern was then invested and processed. Clear denture acrylic resin was packed and heat polymerized using conventional complete denture techniques, deflasked, and polished. A round opening 8-10 mm in diameter was created in the occlusal guard between the maxillary and mandibular teeth to allow access for a suction tube. This design of this occlusal guard offered several advantages over other custom designs and other commercially available products:reflects oral tissue and the tongue from the occlusal table, which may accelerate healing of the traumatized tissues;permits a full range of mandibular motion by using a flat occlusal plane design; allows placement of a suction tube when rinsing and cleaning the mouth without removing and reinserting the prosthesis; is easily fabricated and cleaned using procedures similar for conventional dental occlusal guards and is durable, rigid, resistant to fracture, odorless, and can be used over a long period of time. The possible disadvantages of this occlusal guard are: it requires intraoral impressions, it requires an additional laboratory fee and it involves the risk of possible dislodgement of the occlusal guard during powerful masticating movement.

\section{Conclusion}

A Team support and multidisciplinary approach mainly Prosthodontist(with Oral Surgeon, Pedodontist/ Orthodontist) is essential to treat and provide necessary care to these special patients.Dental services rendered to them needs thorough knowledge, skills, and a suitable hospital set up. "Eyes see only what the mind knows." This kind of patints require a Prosthodontist or practitioner who is well educated, updated as well is brimming with empathy and professional experience.

\section{References}

[1]. E.A.Rovenstine.The incidence, diagnosis and management of coma.Anesthesiology. The journal of American society of Anesthesists.Jan 1945, vol 6, no 1.

[2]. Taira Kobayashi, Henry Ghanem,Koji Umezawa,Junichi Mega, Misao Kawara, Jocelyne S. Feine. Treatment of Self-Inflicted Oral Trauma in a Comatose Patient: A Case Report. J Can Dent Assoc 2005; 71(9):661-4.

[3]. Marianne O'Reilly. Oral care of the critically ill: a review of the literature and guidelines for practice.Australian Critical Care.Volume 16, Issue 3, August 2003, Pages 101-110.

[4]. A. Dougall \& J. Fiske. Access to special care dentistry, part 4. Education.British Dental Journal 205, 119 - 130 (2008)

[5]. Schimmel, M., Schoeni, P., Zulian, G. B. and Müller, F. (2008), Utilisation of dental services in a university hospital palliative and long-term care unit in Geneva. Gerodontology, 25: 107-112.

[6]. Jeff Burgess, Arlen D Meyers.Dental Management in the Medically Compromised Patient.

[7]. Kerrod B. Hallett. Neuropathological chewing: A dental management protocol and treatment appliances for pediatric patients.Special Care in Dentistry.Volume 14, Issue 2, pages 61-64, March 1994.

[8]. Navpreet Chhatwal, Chandrasekharan Nair K.,Jayakar Shetty. Role of Prosthodontists in the oral care of critically ill patients. KDJ Vol.34, No. 1, January 2011.

[9]. E Wayne Davis.Oral injury prevention appliance for comatose patients and the like.

[10]. Hanson,Gerald E., Ogle, Richard G., Giron,Lui.A tongue stent for prevention of oral trauma in the comatose patient.Critical care Medicine.Oct 1975, Vol 3, issue 5.

[11]. Theodore E.D. Peters, Allan E. Blair, Reese G. Freeman. Prevention of self-inflicted trauma in comatose patients.Oral Surgery, Oral Medicine, Oral PathologyVolume 57, Issue 4, April 1984, Pages 367-370.

[12]. Oral Surgery, Oral Medicine, Oral Pathology, Oral Radiology, and Endodontology.Volume 97, Issue 4, April 2004, Pages 471-475.

[13]. W.A. Levine, C.B. Hoek, R.K. Fenster. An occlusal prosthesis to assure airway patency in the comatose patient. Journal of Prosthetic Dentistry.Volume 44, Issue 4 , Pages 451-452, October 1980.

[14]. S.B.Finn.Clinical Pedodontics, $4^{\text {th }}$ edition.W.B.Saunders Co, 1995.

[15]. D.M.Laskin.Oral and Maxillofacial Surgery.vol 1, C V Mosby Co, 1999. 